\title{
Riesgo de desarrollar LIE-AG en pacientes con tamizaje inicial positivo para VPH y citología cervicovaginal negativa en seguimiento hasta 10 años: revisión sistemática y metaanálisis
}

\section{Risk of developing H-SIL in patients with initial positive screening for HPV and negative cervicovaginal cytology in follow-up up to 10 years: systematic review and meta-analysis}

\author{
Lida M. Peña-Amezquita ${ }^{1}$, Alirio R. Bastidas-Goyes ${ }^{2}$, Flavio J. Rosero-Díaz del Castillo", \\ Adriana M. Ramírez-Olaya ${ }^{1}$, Catalina Palacio-Giraldo, Marcos F. Castillo-Zamora ${ }^{1 *}$ y Jairo Amaya-Guio ${ }^{3}$ \\ ${ }^{1}$ Departamento de Ginecología y Obstetricia, Universidad de La Sabana, Chía, Cundinamarca; ${ }^{2}$ Departamento de Epidemiología, Universidad de \\ La Sabana, Chía, Cundinamarca; ${ }^{3}$ Departamento de Ginecología y Obstetricia, Universidad Nacional de Colombia, Bogotá. Colombia
}

\section{Resumen}

Introducción: El cáncer de cérvix es el segundo en frecuencia y el tercero en mortalidad; la infección por el virus del papiloma humano (VPH) está asociada al riesgo de cáncer; sin embargo, no se conoce el riesgo acumulado a 1, 2, 3, 5 y 10 años. Objetivo: Determinar el riesgo de las pacientes VPH positivo con evaluación inicial citológica negativa para desarrollar lesión intraepitelial de alto grado a lo largo del tiempo. Método: Se realizó una revisión sistemática de artículos en inglés y español de los últimos 20 años, usando las bases de datos PubMed, Cochrane, LILACS, ProQuest y Embase. Se incluyeron ensayos clínicos aleatorizados en los que se determinaba el estado VPH y se realizaba seguimiento con citología cervicovaginal a 1, 2, 3, 5 y 10 años en mujeres de 20-64 años. Resultados: Se incluyeron siete ensayos clínicos aleatorizados, con un total de 98.521 mujeres, de ellas $8820 \mathrm{VPH}$ positivo y $89.701 \mathrm{VPH}$ negativo al ingreso, seguidas hasta por 10 años con citología cervicovaginal, encontrando que la infección por VPH es un factor de riesgo para desarrollar lesiones intraepiteliales de alto grado a 2, 5 y 10 años, con un riesgo relativo de 110.94 (79.41-154.97), 83.65 (55.22-126.73) y 29.71 (5.72154.33), respectivamente. Conclusiones: La infección por VPH es un factor de riesgo importante para el desarrollo de lesiones intraepiteliales de alto grado a 2, 5 y 10 años.

Palabras clave: Ensayo controlado aleatorizado. Ensayo clínico controlado. Test DNA del virus del papiloma humano. Test de Papanicolau. Infección por papilomavirus y neoplasia intraepitelial cervical.

\section{Abstract}

Introduction: Cervical cancer is the second in frequency and the third in mortality, infection by the human papillomavirus (HPV) is associated with the risk of increased cancer; however, the cumulative risk of 1, 2, 3, 5 and 10 years is not known. Objective: To determine the risk of HPV-positive patients with negative initial cytological evaluation for developing high-grade intraepithelial lesion over time. Method: A systematic review of articles in English and Spanish in the last 20 years was carried out, using the PubMed, Cochrane, LILACS, ProQuest and Embase databases. Randomized clinical trials were inclu-

Correspondencia:

*Marcos F. Castillo-Zamora

E-mail: marcoscz@unisabana.edu.co

CC BY-NC-ND (https://creativecommons.org/licenses/by-nc-nd/4.0).
Disponible en internet: 07-02-2022 Rev Chil Obstet Ginecol. 2021;86(6):506-515

www.rechog.com 
ded in which HPV was performed and subsequent follow-up with cervicovaginal cytology at 1,2, 3, 5 and 10 years in women aged 20-64 years. Results: Seven randomized clinical trials were included, a total of 98,521 women, 8820 with positive HPV and 89701 negative on admission and followed up for up to 10 years with cervicovaginal cytology. Finding that HPV infection is a risk factor for developing high-grade intraepithelial lesion at 2, 5 and 10 years with a relative risk of 110.94 (79.41-154.97), 83.65 (55.22- 126.73) and 29.71 (5.72-154.33), respectively. Conclusions: HPV infection is an important risk factor for the development of high-grade intraepithelial lesion at 2, 5 and 10 years.

Keywords: Randomized controlled trial. Controlled clinical trial. Human papillomavirus DNA test. Pap test. Papillomavirus infection and cervical intraepithelial neoplasia.

\section{Introducción}

El cáncer de cérvix es el segundo cáncer en frecuencia y el tercero en mortalidad. En el año 2012 se estimaron aproximadamente 527.600 nuevos casos en el mundo, con 265.700 muertes anuales ${ }^{1}$. En los Estados Unidos se estima que 79.000 .000 mujeres entre los 14 y los 59 años se infectan por al menos un genotipo de virus del papiloma humano (VPH) ${ }^{2}$. La carga de esta enfermedad es mayor en los países en vías de desarrollo, donde los programas de tamización son menos eficientes y existen mayores conductas de riesgo; el $85 \%$ de los casos nuevos y el $90 \%$ de la mortalidad ocurren en estos países y los grupos más vulnerables son los de estado socioeconómico bajo ${ }^{3}$.

El cáncer de cérvix es una enfermedad prevenible, la cual resulta de la infección persistente del tracto genital inferior por el VPH. Alrededor de 15 tipos del VPH se consideran de alto riesgo, y los tipos 16 y 18 representan el $71 \%$ de estas infecciones ${ }^{4}$. Solo una décima parte de las infecciones se vuelven persistentes y dan lugar a lesiones precancerosas ${ }^{3}$; de ahí que la detección de la infección por VPH y el seguimiento oportuno deberían disminuir a largo plazo el desarrollo de cáncer cervical hasta ser una enfermedad infrecuente.

Los cambios citológicos generados por el VPH se relacionan con la virulencia del genotipo que infecta; no obstante, hasta cerca del $90 \%$ de las infecciones incidentes por este virus no son detectables en un periodo de 2 años ${ }^{3}$. En las pacientes con VPH positivo y citología cervicovaginal (CCV) negativa podría interpretarse que la infección es por un virus poco agresivo o que la infección es depurada por el sistema inmunitario, y que el riesgo de desarrollar cáncer a largo plazo es bajo; no obstante, el seguimiento habitual de una paciente con VPH positivo y CCV negativa es de 18 a 24 meses, pero no se conoce si este riesgo persiste durante un tiempo más prolongado.

El propósito de esta revisión es determinar el riesgo de las pacientes VPH positivo con CCV inicial negativa para desarrollar lesión intraepitelial de alto grado (LIE-AG) a través del tiempo.

\section{Método}

\section{Tipo de estudio}

Se realizó una revisión sistemática de la literatura y metaanálisis.

\section{Criterios de elegibilidad}

Tipo de estudio: se incluyeron estudios multicéntricos u observacionales y ensayos clínicos aleatorizados que evaluaran el riesgo de desarrollar LIE-AG hasta por 10 años en pacientes con CCV negativa y tamizaje positivo para VPH sin tener en cuenta la tipificación.

- Tipo de participantes: mujeres de 19 a 69 años de edad con tamizaje positivo para VPH y CCV negativa.

- Tipos de intervención: pacientes que mediante tamización para cáncer cervical se hubieran encontrado con VPH positivo y resultado de CCV negativa.

- Tipos de desenlaces: reporte de neoplasia intraepitelial cervical de segundo o tercer grado en el estudio citológico a los 3 y 5 años de seguimiento.

\section{Fuente de datos}

Se realizó una revisión sistemática de artículos en inglés y español en los últimos 20 años usando las bases de datos PubMed, Cochrane, LILACS, ProQuest y Embase.

\section{Búsqueda}

Los términos incluidos fueron: mujer, adulto joven, adulto, test DNA del VPH, test de Papanicolaou, infección por papilomavirus y neoplasia intraepitelial cervical (Apéndice 1). 


\section{Selección de los estudios}

La valoración de la elegibilidad de los estudios se realizó de manera cegada y estandarizada por cuatro revisores de forma independiente, que inicialmente valoraron el título y el resumen para incluir los estudios para lectura completa. Una vez seleccionados los estudios, los revisores, en trabajo independiente y cegado, eligieron los que ingresaron al estudio. Los desacuerdos fueron resueltos por un experto. Los criterios de inclusión para el estudio fueron ensayos controlados aleatorizados, casos y controles, y estudios multicéntricos; adicionalmente, también se incluyeron mujeres de 19-69 años de edad con prueba de tamizaje para VPH positiva y CCV negativa, en idiomas inglés y español. Los criterios de exclusión fueron pacientes gestantes, inmunosuprimidas, con antecedente de histerectomía y con lesiones cervicales previas en seguimiento.

\section{Proceso de recolección de datos}

Se desarrolló una hoja de extracción de datos (basada en la plantilla de extracción de datos del Grupo Cochrane de Consumidores y Comunicación) en la que se incluyeron ocho estudios tras hacer una lectura completa. Los desacuerdos se resolvieron mediante discusión entre los cuatro autores de la revisión; si no se llegaba a un acuerdo, se planeó que un experto decidiera la inclusión del artículo. Finalmente se excluyó un artículo por no contar con datos suficientes para adicionarlos al metaanálisis.

\section{Elementos de datos}

Se extrajo información de cada estudio incluido sobre:

- Características de las participantes del ensayo y los criterios de inclusión y exclusión del ensayo.

- Tipo de intervención, incluyendo si se realizaron al ingreso CCV y prueba de VPH.

- Tipo de medida de resultado en que se evaluó si la tamización para VPH era positiva y la CCV negativa.

\section{Riesgo de sesgo en estudios individuales}

Para determinar la validez de los estudios elegibles, los pares de revisores trabajaron independientemente y determinaron la recolección de datos y los evaluadores de resultados realizados por un experto. El riesgo de sesgo para los estudios incluidos se evaluó con la herramienta ROBIS.

\section{Resultados}

\section{Selección de los estudios}

Se identificaron siete estudios para su inclusión en la revisión. La búsqueda en las bases de datos PubMed, ProQuest, Embase, LILACS y Cochrane proporcionó un total de 170 citas. Después de eliminar duplicados, quedaron 167 estudios. De estos, 161 fueron descartados tras revisar los títulos y resúmenes, ya que no cumplían con el criterio de inclusión «tipo de estudio». El texto completo de los seis artículos restantes se examinó con más detalle, y de estos, un estudio no cumplió con los criterios de inclusión «tipos de intervención» y «tipo de desenlace». Cinco estudios cumplieron los criterios de inclusión y se consideraron en la revisión sistemática. Se identificaron dos estudios que cumplieron con los criterios de inclusión al verificar las referencias de trabajos relevantes y localizados. No se encontraron estudios relevantes no publicados (Fig. 1).

\section{Características de los estudios incluidos}

Se seleccionaron siete estudios, de los cuales seis fueron ensayos clínicos aleatorizados y uno fue un estudio de tamizaje multicéntrico, teniendo como base un ensayo clínico aleatorizado. La duración del seguimiento fue de un año para el $\mathrm{CCCaST}^{5}$ y el HART ${ }^{6}$, de 2 años para el ARTISTIC ${ }^{7}$ y el FOCAL ${ }^{8}$, de 3 años para el NTCC ${ }^{9}$ y el VUSA $^{10}$, de 5 años para el ARTISTIC y el POBASCAM ${ }^{11}$, y de 10 años para el ARTISTIC y el CCCaST.

Los estudios sumaban un total de 114.204 participantes. Los principales criterios de inclusión fueron pacientes de sexo femenino de 30 a 69 años de edad, con tamizaje para VPH positivo, CCV negativa y ensayo clínico aleatorizado.

Se realizó tamizaje para detectar VPH en todos los estudios seleccionados, consistente en la realización del test de VPH y la CCV al ingreso (Fig. 10) y como seguimiento.

En todos los estudios, el resultado primario evaluado fue el desarrollo de LIE-AG, cáncer in situ o carcinoma; dentro de la revisión y el metaanálisis se tuvieron en cuenta las LIE-AG.

\section{Riesgo de sesgo en los estudios}

Se realizaron resúmenes de la calidad metodológica de los estudios incluidos para cada uno de los ítems que se evaluaron en todos los estudios (Fig. 2) y para cada estudio incluido (Fig. 3). 


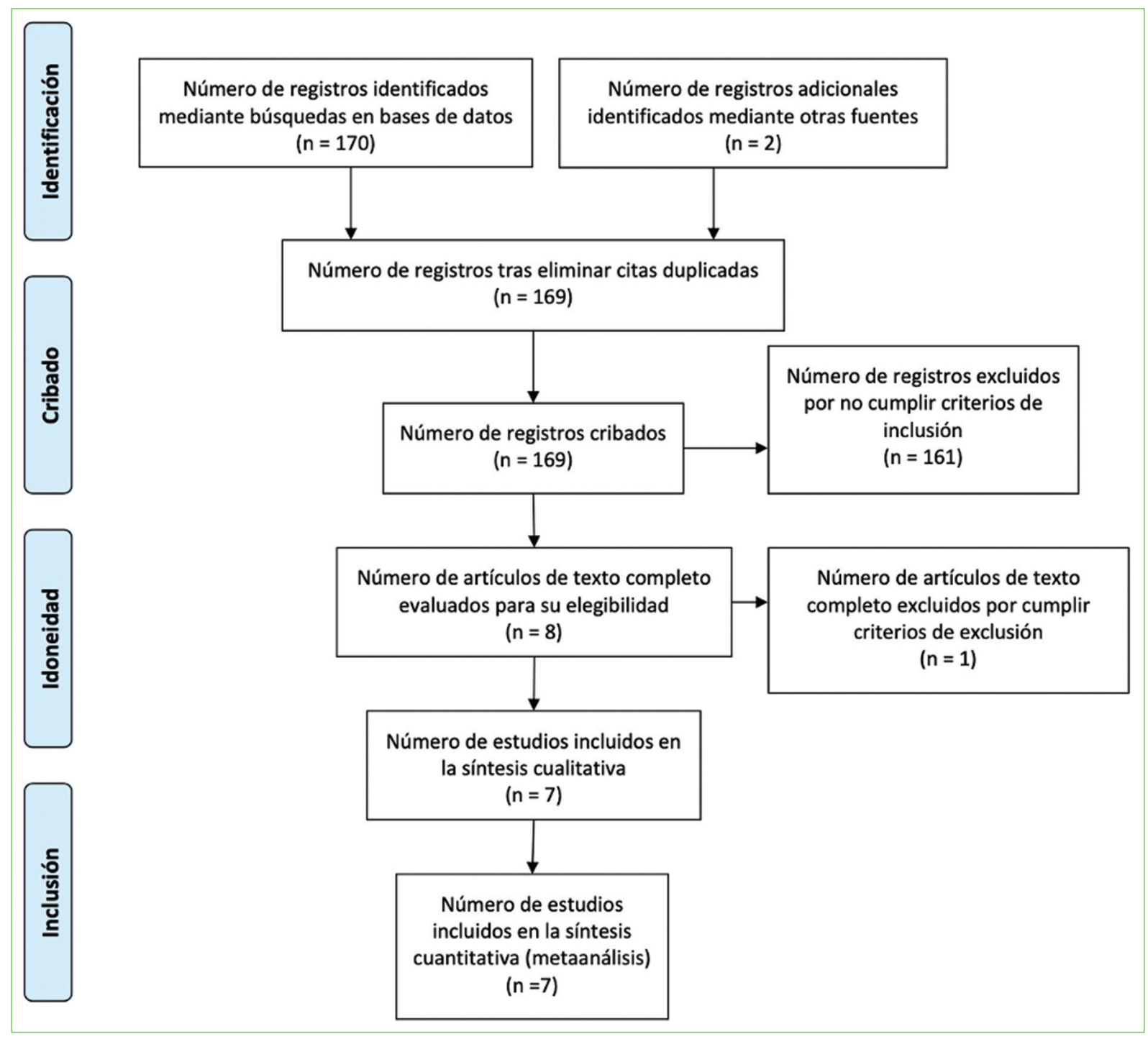

Figura 1. Flujograma del estudio.

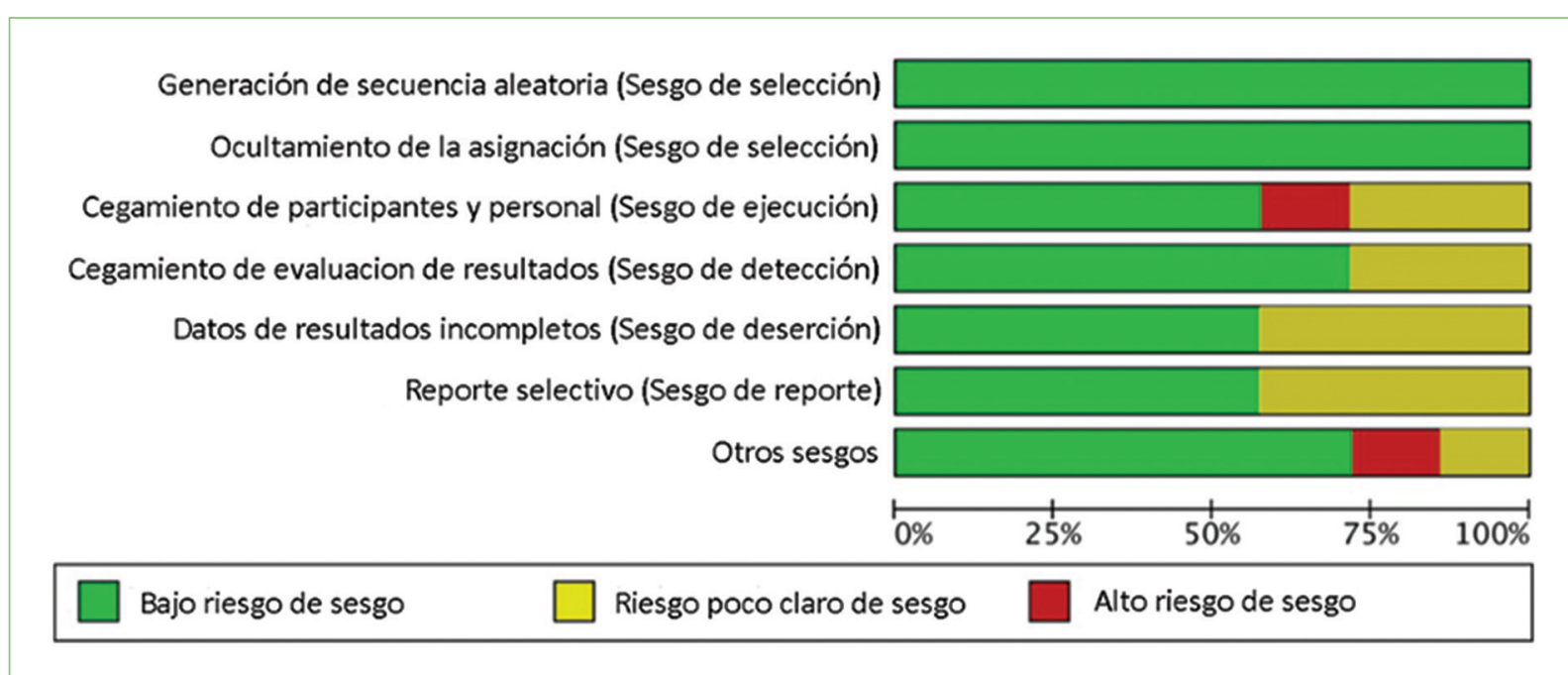

Figura 2. Gráfico de «riesgo de sesgon: juicios de los revisores acerca de cada riesgo de sesgo expresado como porcentaje en todos los estudios incluidos. 


\begin{tabular}{|c|c|c|c|c|c|c|c|}
\hline & 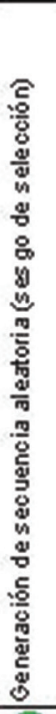 & 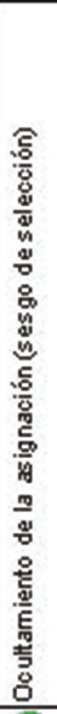 & 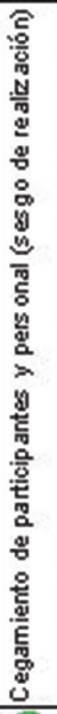 & 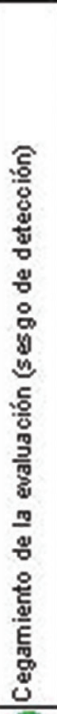 & 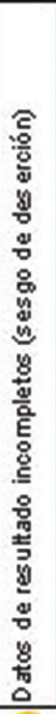 & 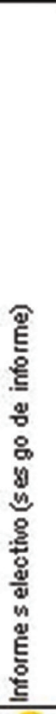 & $\begin{array}{l}\stackrel{8}{\circ} \\
\text { y } \\
\text { n } \\
\stackrel{2}{5} \\
\end{array}$ \\
\hline ARTSTC RCT 2019 & + & + & 4 & + & $?$ & $?$ & 4 \\
\hline CCCAST 2016 & 4 & 4 & 4 & 4 & + & + & $?$ \\
\hline FOCAL 2018 & 4 & + & $?$ & + & $?$ & $(+)$ & + \\
\hline HART 2003 & 4 & + & $?$ & $?$ & + & $?$ & 8 \\
\hline NTCC 2015 & + & + & + & $?$ & $?$ & + & $(+)$ \\
\hline POBASCAM 2007 & + & + & 4 & + & + & + & + \\
\hline VUSA 2012 & 4 & + & $\theta$ & + & + & $?$ & 4 \\
\hline
\end{tabular}

Figura 3. Resumen del "riesgo de sesgo»: juicios de los revisores sobre cada elemento de riesgo de sesgo de cada estudio incluido.

\section{Resultados de los estudios individuales}

En el seguimiento a 1 año se halló un riesgo relativo (RR) de 1,3, con un intervalo de confianza del 95\% (IC95\%) de 0.72-2.34 y un I ${ }^{2}$ del 0\% (Fig. 4); a 2 años el RR es de 110.94, con un IC95\% de 79.41-154.97 y un $I^{2}$ del $0 \%$ (Fig. 5); a 3 años el RR es de 6.68, con un IC95\% de $0.47-95.31$ y un $\mathrm{I}^{2}$ del $99 \%$ (Fig. 6); a 5 años el RR es de 83.65 , con un IC95\% de 55.22-126.73 y un $\mathrm{I}^{2}$ del30\% (Fig. 7); y a 10 años el RR es de 29.71, con un IC95\% de $5.72-154.33$ y un $\mathrm{I}^{2}$ del 67\% (Fig. 8).

\section{Fondos de financiación de los estudios incluilos}

De los siete artículos seleccionados, CCCAST y POBASCAM no fueron financiados por fuentes externas. Los demás sí tuvieron fuente de financiación, pero los financiadores no formaron parte del diseño del estudio, la recopilación de los datos, el análisis de los datos, la interpretación de los datos ni la redacción del informe, y tampoco de la decisión de enviarlo para su publicación.

\section{Discusión}

En nuestro estudio se pretende determinar el riesgo de desarrollar una LIE-AG en aquellas pacientes que, teniendo infección por VPH, no presentan lesión cervical evidente en la CCV por fuera de los tiempos esperados de depuración del virus. Se revisaron artículos con seguimiento de las pacientes a 1, 2, 3, 5 y 10 años. Se encontró un aumento del riesgo para desarrollo de LIE-AG hasta los 10 años de seguimiento en las pacientes con infección por VPH y CCV negativa.

En dos ensayos dentro de la revisión sistemática CCCAST y HART, los cuales realizaron seguimiento a 12 meses de las pacientes, se logró establecer que la infección por VPH no implica un riesgo de desarrollar cáncer, puesto que en este tiempo la sola presencia del virus no es suficiente para desarrollar una LIE-AG.

Se ha documentado que cerca del $90 \%$ de las primoinfecciones por VPH no son detectables en un periodo de 2 años desde el momento de la infección y solo persisten en una pequeña proporción. Únicamente una décima parte de todas las infecciones se vuelven persistentes y son estas mujeres las pueden desarrollar lesiones cervicales precancerosas ${ }^{2,3}$. Tal información es acorde con lo encontrado en nuestra revisión. En dos ensayos, ARTISTIC y FOCAL, con seguimiento a 2 años, se evidenció que después de realizada la prueba de tamizaje con VPH positivo se eleva el riesgo para desarrollar LIE-AG tanto en pacientes con CCV alterada como en aquellas con CCV negativa; en otras palabras, no es esperable que una paciente con VPH positivo y CCV negativa desarrolle una LIE-AG a 2 años.

Con respecto al seguimiento a 3 años de los estudios NTCC y VUSA, si bien al revisar en conjunto los datos no se encuentra una significancia estadística debido en parte a la amplia heterogeneidad de los resultados (Fig. 9), la lectura crítica individual de cada estudio refleja que el riesgo de desarrollar LIE-AG persiste en el tiempo. Si se tiene en cuenta que la incidencia de LIE-AG es muy baja dentro de los 3 años posteriores a una CCV normal (10 a 66 por 10.000) $)^{12}$, y que el riesgo de muerte durante toda la vida atribuido al cáncer cervical es de 0.05 por 1000 con tamizaje citológico cada 3 años ${ }^{13}$, se podría inferir entonces la necesidad de observar al grupo de pacientes con prueba de VPH positiva y CCV negativa siempre y cuando el seguimiento no muestre alteraciones celulares. No obstante, los factores que determinan qué infección por VPH será persistente no se conocen con certeza. El genotipo del VPH es el factor más determinante para la persistencia y la progresión de la enfermedad. EI VPH 


\begin{tabular}{|c|c|c|c|c|c|c|c|c|c|c|}
\hline Estudio o subgrupo & $\begin{array}{r}\mathrm{HPV}+ \\
\text { Eventos }\end{array}$ & Total & $\begin{array}{l}\text { HPV - } \\
\text { Eventos }\end{array}$ & Total & Peso & $\begin{array}{l}\text { Riesgo Relativo } \\
\text { IV, Aleatorizado, } 95 \% \mathrm{CI}\end{array}$ & & $\begin{array}{l}\text { Riesgo Relativo } \\
\text { IV, Aleatorizado, } 95 \% \mathrm{Cl}\end{array}$ & & \\
\hline CCCAST 2016 & 32 & 67 & 4 & 14 & $46.0 \%$ & $1.67[0.70,3.97]$ & & & & \\
\hline HART 2003 & 21 & 90 & 6 & 27 & $54.0 \%$ & $1.05[0.47,2.33]$ & & & & \\
\hline Total $(95 \% \mathrm{Cl})$ & & 157 & & 41 & $100.0 \%$ & $1.30[0.72,2.34]$ & & & & \\
\hline Eventos totales & 53 & & 10 & & & & & & & \\
\hline \multicolumn{11}{|c|}{ Heterogeneidad: $\mathrm{Tau}^{2}=0.00 ; \mathrm{Chi}^{2}=0.60, \mathrm{df}=1(P=0.44) ; \mathrm{I}^{2}=0 \%$} \\
\hline \multicolumn{7}{|c|}{ Test for overall effect: $Z=0.88(P=0.38)$} & 0.01 & HPV - HPV + & 10 & 100 \\
\hline
\end{tabular}

Figura 4. Forest plot con riesgo relativo e intervalo de confianza del 95\% para seguimiento a 1 año para desarrollo de lesión intraepitelial de alto grado.

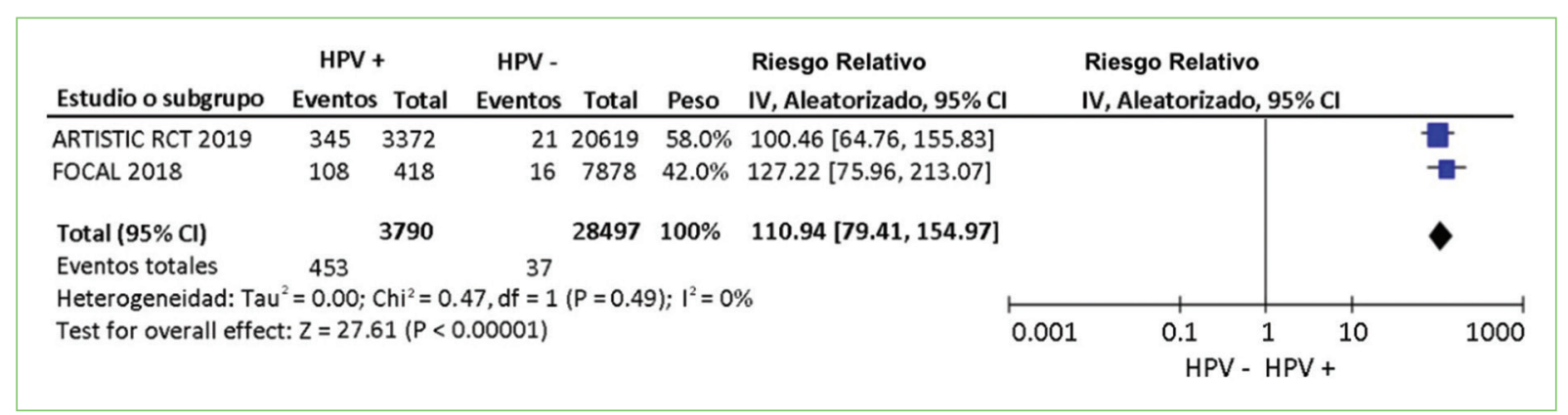

Figura 5. Forest plot con riesgo relativo e intervalo de confianza del 95\% para seguimiento a 2 años para desarrollo de lesión intraepitelial de alto grado.

\begin{tabular}{|c|c|c|c|c|c|c|c|c|c|c|}
\hline Estudio o subgrupo & $\begin{array}{r}\mathrm{HPV}+ \\
\text { Eventos }\end{array}$ & Total & $\begin{array}{l}\text { HPV - } \\
\text { Eventos }\end{array}$ & Total & Peso & $\begin{array}{l}\text { Riesgo Relativo } \\
\text { IV, Aleatorizado, } 95 \% \mathrm{Cl}\end{array}$ & \multicolumn{3}{|c|}{$\begin{array}{l}\text { Riesgo Relativo } \\
\text { IV, Aleatorizado, } 95 \% \mathrm{Cl}\end{array}$} & \\
\hline NTCC 2015 & 110 & 866 & 29 & 395 & $50.2 \%$ & $1.73[1.17,2.56]$ & & & \multirow{2}{*}{\multicolumn{2}{|c|}{$\rightarrow$}} \\
\hline VUSA 2012 & 139 & 1021 & 16 & 3063 & $49.8 \%$ & $26.06[15.61,43.51]$ & & & & \\
\hline Total $(95 \% \mathrm{Cl})$ & & 1887 & & 3458 & $100.0 \%$ & $6.68[0.47,95.31]$ & & & & \\
\hline Eventos totales & 249 & & 45 & & & & & & & \\
\hline \multicolumn{7}{|c|}{ Heterogeneidad: $\mathrm{Tau}^{2}=3.62 ; \mathrm{Chi}^{2}=67.94, \mathrm{df}=1(\mathrm{P}<0.00001) ; \mathrm{I}^{2}=99 \%$} & 0.001 & 0.1 & 110 & 1000 \\
\hline \multicolumn{7}{|c|}{ Test for overall effect: $Z=1.40(P=0.16)$} & \multicolumn{4}{|c|}{ HPV - HPV + } \\
\hline
\end{tabular}

Figura 6. Forest plot con riesgo relativo e intervalo de confianza del 95\% para seguimiento a 3 años para desarrollo de lesión intraepitelial de alto grado.

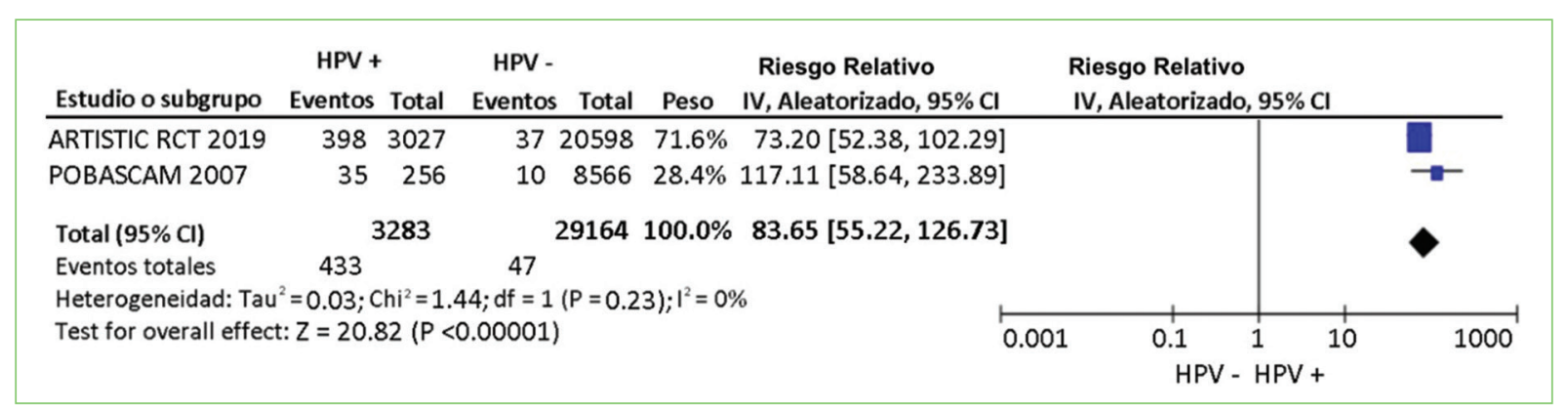

Figura 7. Forest plot con riesgo relativo e intervalo de confianza del $95 \%$ para seguimiento a 5 años para desarrollo de lesión intraepitelial de alto grado. 


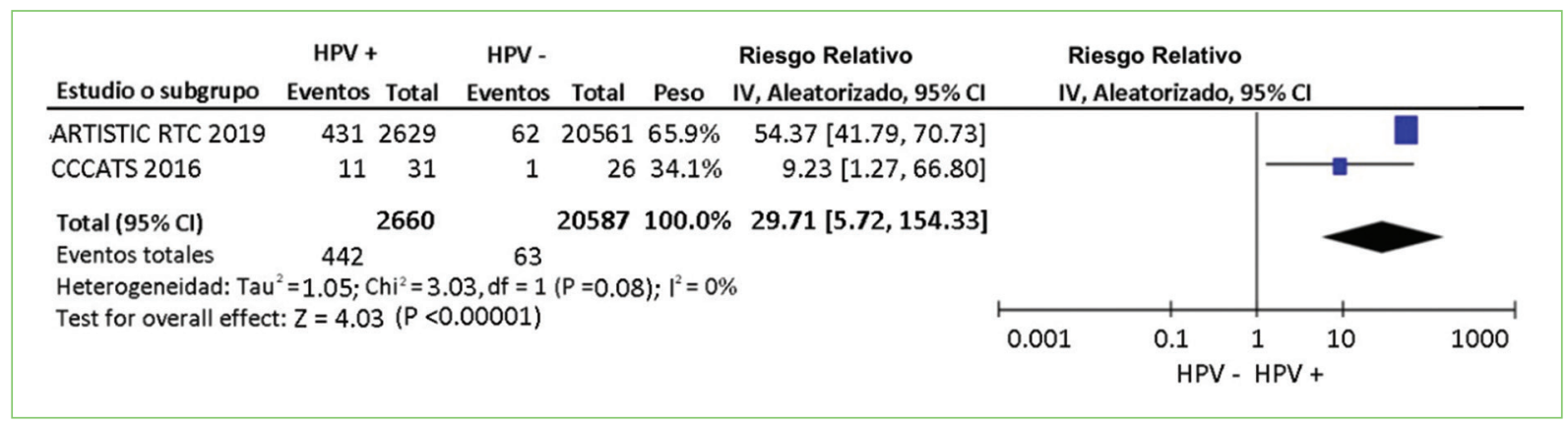

Figura 8. Forest plot con riesgo relativo e intervalo de confianza del 95\% para seguimiento a 10 años para desarrollo de lesión intraepitelial de alto grado.

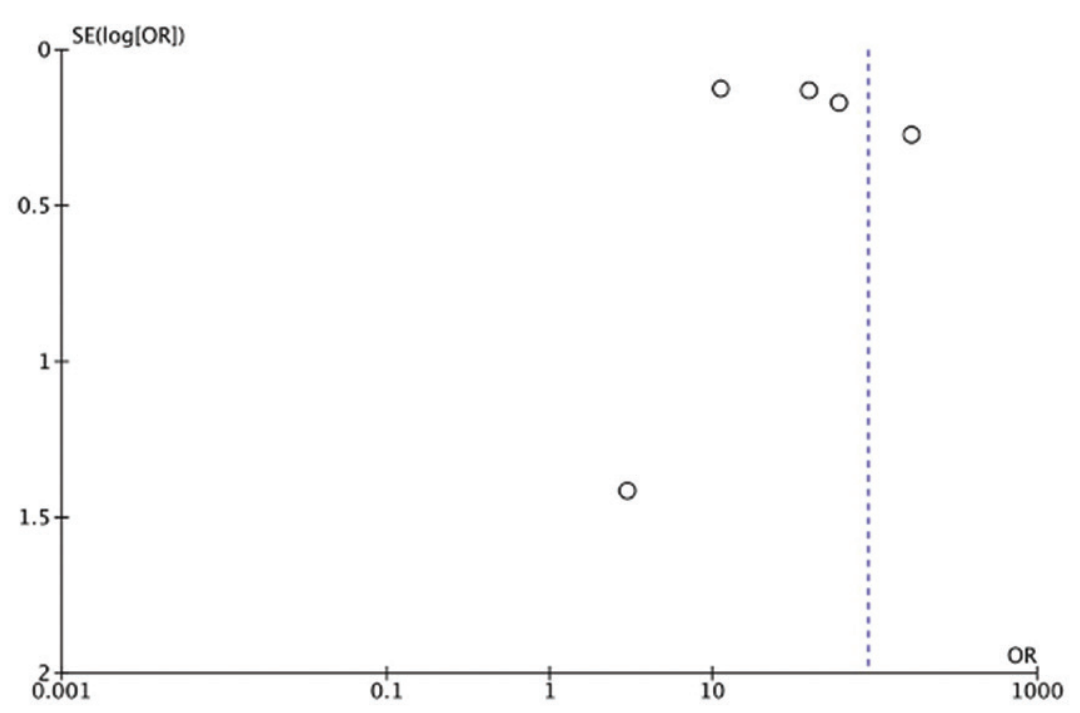

Figura 9. Funnel plot de los diferentes estudios incluidos.

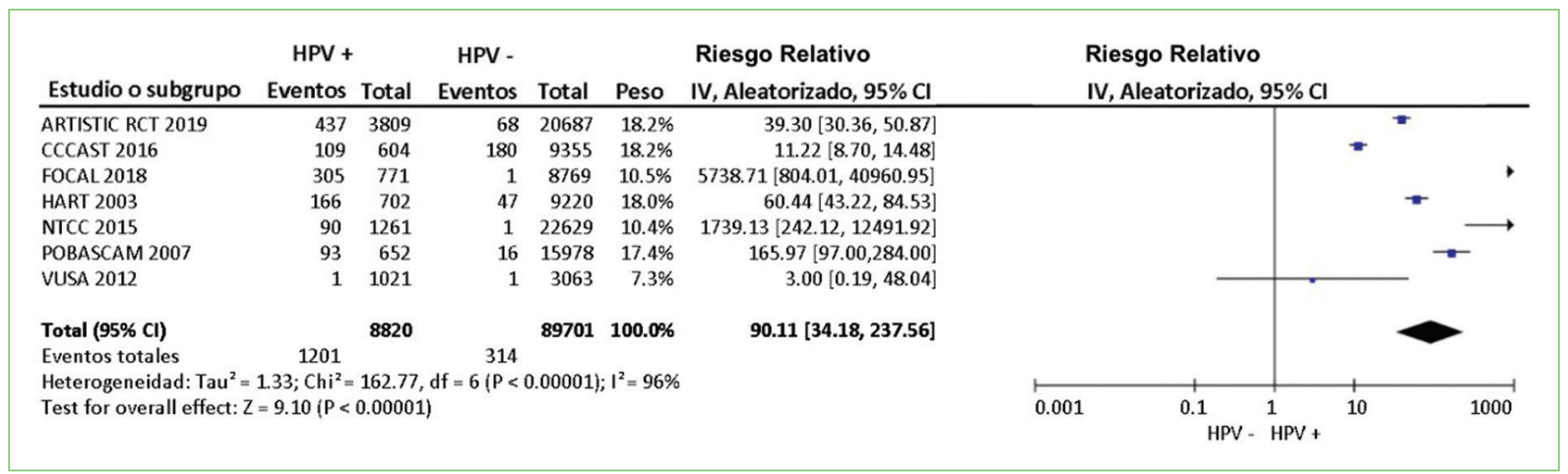

Figura 10. Estado de ingreso de las pacientes.

16 tiene el mayor potencial carcinogénico y representa entre el $55 \%$ y el $60 \%$ de todos los casos de cáncer en el mundo, seguido por el VPH 18, que responde por el $10 \%$ al $15 \%$ de los casos $^{14}$.
Seguimientos a más largo plazo, como los reportados por los estudios ARTISTIC y POBASCAM a 5 años, y CCCAST y ARTISTIC a 10 años, son congruentes con la información obtenida de estudios a menor término, que 
muestran que la infección es determinante para el desarrollo de la enfermedad. Estos seguimientos prolongados hacen pensar que el incremento en los casos positivos de VPH se deben a que a mayor edad, menor posibilidad de depuración del virus especialmente en mujeres mayores de 30 años, como refieren Chen et al.15 en su estudio, en el que se demuestra que el riesgo acumulado de cáncer de cuello uterino aumenta con la edad, siendo del $5,5 \%$, el $14,4 \%$ y el $18,1 \%$ para las mujeres de $30-44$ años, $45-54$ años y 55 años o más, respectivamente.

Por último, la evidencia obtenida en este estudio está ampliamente a favor del aumento del riesgo de desarrollar LIE-AG ante la presencia de infección por VPH sin alteraciones citológicas, en especial con seguimiento a los 2, 5 y 10 años. Este resultado plantea la necesidad de desarrollar seguimientos especiales para las pacientes con prueba de VPH positiva y CCV negativa, similares a los existentes en pacientes con VPH positivo y alteración citológica.

\section{Limitaciones}

Dentro de los resultados de los estudios revisados no se aportan datos totales del tipo de VPH que se tipificó, ni de la persistencia del virus en las pacientes seguidas; sin embargo, este metaanálisis combinó los datos de siete ensayos clínicos controlados aleatorizados de pacientes que fueron llevadas a detección de VPH con posterior seguimiento con CCV. La limitación de no tipificar el VPH puede convertirse en fuente de nuevas investigaciones para diferenciar el riesgo por los genotipos 16 y 18, por su alta prevalencia y alto poder oncogénico, y compararlo con el de otros genotipos de alto riesgo, en pacientes con VPH positivo y CCV negativa, situación que puede influir en los protocolos de manejo y de seguimiento.

\section{Conclusiones}

Esta revisión sistemática con metaanálisis permite concluir con datos estadísticamente significativos que la infección por el VPH detectada por una prueba de VPH es un factor de riesgo importante para el desarrollo de LIE-AG a 2, 5 y 10 años en pacientes con CCV negativa. Este resultado plantea la necesidad de desarrollar seguimientos especiales para las pacientes con prueba de VPH positiva y CCV negativa similares a los existentes para pacientes con VPH positivo y alteración citológica.

\section{Financiamiento}

El artículo se realizó con recursos propios.

\section{Conflicto de intereses}

Los autores declaran no tener conflictos de intereses.

\section{Responsabilidades éticas}

Protección de personas y animales. Los autores declaran que para esta investigación no se han realizado experimentos en seres humanos ni en animales.

Confidencialidad de los datos. Los autores declaran que en este artículo no aparecen datos de pacientes.

Derecho a la privacidad y consentimiento informado. Los autores declaran que en este artículo no aparecen datos de pacientes.

\section{Bibliografía}

1. Ferlay J, Soerjomataram I, Ervik M, Dikshit R, Eser S, Mathers C, et al. GLOBOCAN 2012: Estimated Cancer Incidence, Mortality and Prevalence Worldwide in 2012 v1.0. IARC CancerBase No. 11. Lyon, France: International Agency for Research on Cancer; 2013.

2. Syrjänen K, Hakama M, Saarikoski S, Väyrynen M, Yliskoski M, Syrjänen S, et al. Prevalence, incidence, and estimated life-time risk of cervical human papillomavirus infections in a nonselected Finnish female population. Sex Transm Dis. 1990;17:15-9.

3. Bhatla N, Aoki D, Sharma DN, Sankaranarayanan R. Cancer of the cervix uteri. Int J Gynaecol Obstet. 2018;143 (Suppl 2):22-36.

4. IARC Working Group. Human papillomaviruses: IARC Monographs on the Evaluation of Carcinogenic Risks to Humans. Lyon: International Agency for Research on Cancer; 2007: 90.

5. Isidean SD, Mayrand MH, Ramanakumar AV, Gilbert L, Reid SL, Rodrigues I, et al. Human papillomavirus testing versus cytology in primary cervical cancer screening: end-of-study and extended follow-up results from the Canadian cervical cancer screening trial. Int J Cancer. 2016;139:2456-66.

6. Cuzick J, Szarewski A, Cubie H, Hulman G, Kitchener H, Luesley D, et al. Management of women who test positive for high-risk types of human papillomavirus: the HART study. Lancet. 2003;362:1871-6.

7. Gilham C, Sargent A, Kitchener HC, Peto J. HPV testing compared with routine cytology in cervical screening: long-term follow-up of ARTISTIC RCT. Health Technol Assess. 2019;23:1-44.

8. Ogilvie GS, van Niekerk D, Krajden M, Smith LW, Cook D, Gondara L, et al. Effect of screening with primary cervical HPV testing vs cytology testing on high-grade cervical intraepithelial neoplasia at 48 months: the HPV FOCAL randomized clinical trial. JAMA. 2018;320:43-52.

9. Bergeron C, Giorgi-Rossi P, Cas F, Schiboni ML, Ghiringhello B, Dalla Palma $P$, et al. Informed cytology for triaging HPV-positive women: substudy nested in the NTCC randomized controlled trial. J Natl Cancer Inst. 2015;107:dju423.

10. Rijkaart DC, Berkhof J, van Kemenade FJ, Coupe VM, Rozendaal L, Heideman DA, et al. HPV DNA testing in population-based cervical screening (VUSA-Screen study): results and implications. Br J Cancer. 2012;106:975-81.

11. Bulkmans NW, Berkhof J, Rozendaal L, van Kemenade FJ, Boeke AJ, Bulk S, et al. Human papillomavirus DNA testing for the detection of cervical intraepithelial neoplasia grade 3 and cancer: 5 -year follow-up of a randomised controlled implementation trial. Lancet. 2007;370:1764-72.

12. Sawaya GF, Kerlikowske K, Lee NC, Gildengorin G, Washington AE. Frequency of cervical smear abnormalities within 3 years of normal cytology. Obstet Gynecol. 2000;96:219-23.

13. Saslow D, Solomon D, Lawson HW, Killackey M, Kulasingam SL, Cain J, et al. American Cancer Society, American Society for Colposcopy and Cervical Pathology, and American Society for Clinical Pathology screening guidelines for the prevention and early detection of cervical cancer. CA Cancer J Clin. 2012;62:147-72.

14. de Sanjosé S, Quint WG, Alemany L, Geraets DT, Klaustermeier JE, Lloveras $B$, et al. Human papillomavirus genotype attribution in invasive cervical cancer: a retrospective cross-sectional worldwide study. Lancet Oncol. 2010;11:1048-56.

15. Chen HC, Schiffman M, Lin CY, Pan MH, You SL, Chuang LC, et al. Persistence of type-specific human papillomavirus infection and increased long-term risk of cervical cancer. J Natl Cancer Inst. 2011;103:1387-96. 


\section{Apéndice 1}

\section{En PubMed}

$\begin{array}{ll}\text { 1. } & \text { Women } \\ \text { 2. } & \text { Adult } \\ \text { 3. } & \text { Young adult } \\ \text { 4. } & \text { DNA Probes, HPV } \\ \text { 5. } & \text { Human Papillomavirus DNA Test } \\ \text { 6. } & \text { Papillomaviridae } \\ \text { 7. } & \text { Papillomavirus Infections } \\ \text { 8. } & 1 \text { or } 2 \text { or } 3 \text { or } 4 \text { or } 5 \text { or } 6 \text { or } 7 \\ \text { 9. } & \text { Citology } \\ \text { 10. } & \text { Papanicolaou Test } \\ \text { 11. } & \text { Vaginal Smears } \\ \text { 12. } & 10 \text { or } 11 \\ \text { 13. } & \text { Cervical Intraepithelial Neoplasia } \\ \text { 14. } & 8 \text { and } 12 \text { and } 13\end{array}$

\section{En Cochrane}

1. Female

2. Young Adult 19-24 years

3. Adult

4. Human Papilloma Virus Infection

5. 1 or 2 or 3 or 4

6. Sampling Of Vagina For Papanicolaou Smear

7. Cervical Intraepithelial Neoplasia

8. 5 and 6 and 7

\section{En Embase}

1. Female

2. Females

3. Woman

4. Women

5. Women, qorking

6. Young adult

7. $\quad$ Adult, young

8. Prime adult

9. Prime adults

10. Yound adult

11. Young adults

12. Adult

13. Adults

14. Grown-ups

15. Grownup

16. Grownups

17. Papillomavirus infection

18. Human papillomavirus infection

19. Papillomavirus infections
20. Papillomaviral infection

21. DNA probe

22. cDNA probes, HPV

23. Probe, DNA

24. Deoxyribonucleic acid probe

25. DNA probes

26. DNA probes, HLA, Probe, DNA

27. 1 or 2 or 3 or 4 or 5 or 6 or 7 or 8 or 9 or 10 or 11 or 12 or 13 or 14 or 15 or 16 or 17 or 18 or 19 or 20 or 21 or 22 or 23 or 24 or 25 or 26

28. Uterine cervix cytology

29. Automated cervical smear

30. Cervical cytology

31. Cervical smear

32. Cervicovaginal smear

33. Cervix cytology

34. Cervix cytology, uterine

35. Cervix smear

36. Cervix uteri smear

37. Cervix uteri, smear

38. Cytology, cervix uteri

39. Smear, cervical

40. uc cervical

41. uterine cervical smear

42. Uterine cervix cytology

43. Uterine cervix smear

44. Uterine endocervix smear

45. Uterus cervix cytology

46. Papanicolaou test

47. Pap smear

48. Papanicolaou test

49. Pap stain

50. Pap test

51. Papanicolaou cytology

52. Papanicolaou method

53. Papanicolaou smear

54. Papanicolaou stain

55. Vagina smear

56. Smear, vaginal

57. Vagina smear

58. Vaginal smear

59. Vaginal smears

60. 28 or 29 or 30 or 31 or 32 or 33 or 34 or 35 or 36 or 37 or 38 or 39 or 40 or 41 or 42 or 43 or 44 or 45 or 46 or 47 or 48 or 49 or 50 or 51 or 52 or 53 or 54 or 55 or 56 or 57 or 58 or 59

61. Uterine cervix carcinoma in situ

62. Cervical intraepithelial neoplasia 2 63. Cervical intraepithelial neoplasia grade 2

64. 61 or 62 or 63 
65. Case control study

66. Case control study

67. Case-control studies

68. Case-control study

69. Control study, case

70. Matched case control

71. Matched case control studies

72. Matched case control study

73. Cohort analysis

74. Analysis, cohort

75. Cohort analysis

76. Cohort fertility

77. Cohort life cycle

78. Cohort studies

79. Cohort study

80. Fertility, cohort

81. Multicenter study

82. Multi-center study

83. Multi-center trial

84. Multi-centre study

85. Multi-centre trial

86. Multicenter study
87. Multicenter trial

88. Multicentre study

89. Multicentre trial

90. Study, multicenter

91. Trial, multicenter

92. Observational study

93. Non experimental studies

94. Non experimental study

95. Nonexperimental studies

96. Nonexperimental study

97. Observation studies

98. Observation study

99. Observational studies

100. Observational studies as topic

101. Observational study

102. Observational study as topic

103. 65 or 66 or 67 or 68 or 69 or 70 or 71 or 72 or 73 or 74 or 75 or 76 or 77 or 78 or 79 or 80 or 1 or 82 or 83 or 84 or 85 or 86 or 87 or 88 or 89 or 90 or 91 or 92 or 93 or 94 or 95 or 96 or 97 or 98 or 99 or 100 or 101 or 102

104. 27 and 60 and 103 